\title{
Interfaces
}

\section{Des textes à toucher du doigt : Rimbaud, Claudel, Ramuz, Carlo Levi}

\section{Michel Arouimi}

\section{(2) OpenEdition}

1 Journals

Édition électronique

URL : http://journals.openedition.org/interfaces/238

DOI : 10.4000/interfaces.238

ISSN : 2647-6754

Éditeur :

Université de Bourgogne, Université de Paris, College of the Holy Cross

\section{Édition imprimée}

Date de publication : 1 janvier 2015

Pagination : 173-185

ISBN : 9780983175247

ISSN : 1164-6225

\section{Référence électronique}

Michel Arouimi, «Des textes à toucher du doigt : Rimbaud, Claudel, Ramuz, Carlo Levi », Interfaces [En ligne], 36 | 2015, mis en ligne le 01 janvier 2018, consulté le 07 janvier 2021. URL : http://

journals.openedition.org/interfaces/238; DOI : https://doi.org/10.4000/interfaces.238

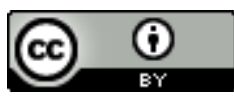

Les contenus de la revue Interfaces sont mis à disposition selon les termes de la Licence Creative Commons Attribution 4.0 International. 


\title{
DES TEXTES A TOUCHER DU DOIGT : RIMBAUD, CLAUDEL, RAMUZ, CARLO LEVI
}

\author{
Michel Arouimi
}

Comprendre un texte sans qu'on le lise est une idée de Claudel, enclin à accorder aux mots ou à la page imprimée, des vertus picturales qui ne se limitent pas aux ressources habituelles de la typographie. Ce credo poétique, malgré son humour apparent, exprime une réalité méconnue, qui n'est pas que celle des textes de Claudel. En exprimant sa dette de poète lyrique à Rimbaud, maître des « rythmes instinctifs », Claudel masquerait en fait l'amplification, dans sa vision des Idéogrammes occidentaux (titre d'un essai bref de 1926), des aspects les plus secrets du projet du Rimbaud «voyant». Les commentateurs voient dans le silence de Rimbaud l'aveu de l'échec de ce projet. Je vais pourtant montrer que ses œuvres sont en adéquation avec ce dernier, et si silence il $\mathrm{y}$ a, la raison en reste à chercher.

Les ambitions du « voyant », dominées par l'idée d'un poème à « voir », imposent l'idée d'un texte qui parlerait à " tous les sens », à commencer par la vue, comme le confirme la récurrence du verbe « voir » dans la célèbre lettre du 15 mai 1871, seconde lettre dite du « voyant ». Or, ce qui est « vu » ne vaut que par sa fonction représentative à l'égard d'une certaine idée de la totalité, dont les critiques modernes nient obstinément le sens spirituel.

L'Illumination Veillées, avec ses trois sections numérotées, est l'exemple le plus frappant de cette aptitude poétique. La première, où domine le thème de l'air : «C'est le repos éclairé [...] L'air et le monde point cherchés. La vie ${ }^{1}$, se présente comme une suite de six alinéas très brefs, parfois ponctués de phrases nominales. Ces dispositions semblent mimer la légèreté de l'air ou le repos. La section II, sur le manuscrit de Rimbaud, est écrite avec une plume plus lourde, comme si l'épaisseur de l'encre mimait celle de la «muraille en face du veilleur», décrite comme " une succession psychologique de coupes de frises, de bandes atmosphériques et d'accidences géologiques. — »... Après ce dernier mot de la phrase, « géologiques », le tiret qui suit le point introduit une phrase nominale de dix-huit mots, qui referme cette section II. Ce tiret semble figurer ces « accidences géologiques » dans l'espace uni de ce paragraphe, tassé comme les pierres imaginables de cette muraille.

Y a-t-il un rapport entre ce mimétisme littéraire qui nous fait voir et sentir l'air, la terre (et l'eau dans la section III), et le mimétisme interhumain, thématisé à la fin de la section II, avec les « groupes sentimentaux avec tous les êtres de tous les caractères parmi toutes les apparences »?

1 Rimbaud, CEuvres Complètes, Bibliothèque de la Pléiade, 2009, pp. 304-5. 
L'apparence régulière des trois premiers aliénas de Veillées III, avant la longue série de points qui les sépare de la dernière phrase, articulée par le signe des deux points, s'accorde avec la vision des " vagues » de la «mer », elles-mêmes incarnées par les " seins d'Amélie », où la critique a reconnu l'anagramme de «L'aimée » de la section I : «Les lampes et les tapis de la veillée font le bruit des vagues [...]. / La mer de la veillée, telle que les seins d'Amélie. »

Les premiers mots de cette section III : «Les lampes », et dans le quatrième et dernier alinéa, l'image du « foyer noir, de réels soleils des grèves », superposent le feu au thème aquatique. Est-ce le feu ou l'eau que mime l'irrégularité visuelle des alinéas de cette section ? Alchimie oblige, ces détails complètent les thèmes de l'air et de la muraille (sections I et II). Et sans doute les sonorités privilégiées dans chaque section évoquent-elles ces éléments naturels, encore mimés par l'espace graphique, et qui se donnent à voir avant même qu'on lise le poème.

J'ai montré ailleurs le système d'échos lexical qui, dans les poèmes de sept strophes Michel et Christine et Plates-bandes d'amarantes, traduit littéralement le « railway » ou le « chemin de fer », deux motifs qui s'inscrivent exactement au milieu de ces deux poèmes. Les détails qui accompagnent ces motifs imprégnés de la violence la plus moderne, sont pourtant les signes (ludique dans le second poème, grave dans le premier) d'une préoccupation métaphysique, impliquant la transcendance : le « Seigneur », dans Michel et Christine, et les « mille diables bleus » dans Plates-bandes d'amarante.

Le chemin de fer vaut dans ces deux poèmes ce que les « crotales » et les « constrictors » valent dans l'espace mouvementé du venimeux poème adressé à Banville, Ce que l'on dit au poète à propos de fleurs. Dans ce poème en vers de cinq sections, les mentions de ces serpents délimitent des séries équivalentes de strophes. Après les douze premières strophes, une allusion aux " crotales » délimite une suite de six strophes avec «Des constrictors d'un hexamètre $»^{2}$. Ce dernier mot révèle le mètre favorisé par la plume de Rimbaud, dès la première section dont les six strophes préparent l'œil à repérer, en II, 6 et III, 6, deux enjambements tout aussi spectaculaires que celui qui divise l'espace harmonisé de Michel et Christine: "L'Ode Açoka cadre avec la / Strophe en fenêtre de Lorette » et « Toi-même, assis là-bas, dans une / Cabane de bambous »...

L'enjambement, comme figuration des reptations musculaires d'un reptile ? Il en irait de même pour la simple inscription des tirets, répartis dans une harmonie mouvante : neuf tirets s'étagent en début de vers, et seulement huit à l'intérieur des vers ${ }^{3}$.

Ibid., pp.149-54.

3 En IV, 10, les deux tirets qui encadrent le mot « fameuses » qui termine un vers (« Des fleurs presque pierre, fameuses ! - ) remédient à ce déséquilibre. Le tiret qui ponctue deux autres vers (en III, 2 et III, 6) ajoute au dynamisme de cette harmonie. 
Le texte du poème s'offre ainsi comme une représentation graphique des serpents auxquels s'identifie le signataire, «Alcide Bava ». Inscrites sous ce pseudonyme, les initiales «A. R. » semblent figurer les deux dents d'un crotale, dont la queue serait représentée par l'anaphore de l'expression « Le $[\mathrm{s}] \mathrm{Ly}[\mathrm{s}]$ », dans les trois premières strophes où l'œil repère encore trois mentions du ou des « lys » avec minuscule à l'initiale, etc.

Ce tête-bêche où la queue du poème figurerait la tête du serpent, peut se mettre en rapport avec les enjeux philosophiques des deux inscriptions de l'adverbe d'affirmation en II, 9 : « Oui, vos bavures de pipeaux » et en IV, 10 : «Oui, trouve au cœur des noirs filons », qui encadrent, symétriquement, ces vers qui se suivent en III, 7 (fin de section) : « raisons / Non moins risibles » et en IV, 1 : « Dis, non les pampas »... La tension du « oui » et du « non », si elle peut passer pour la clé de l' " alchimie du verbe », n'en révèle pas moins le tourment si obscur (« l'inconnu » dont parle le « voyant»?) qui inspire peut-être cette concurrence mimétique du texte avec le réel.

Non moins visibles, des majuscules distinguant de nombreux mots à l'intérieur des vers. Certaines désignations qui regroupent deux mots permettent de réduire cet ensemble (« Violette du Bois », «L'Ode Açoka », « Sombres Indulgences », « Bleus Thyrses », « Monsieur Figuier », « Monsieur Hachette »). Le nombre obtenu correspond alors à la moitié de celui des vers du poème, vers dont le premier mot a bien sûr une majuscule à l'initiale. On ne saurait traduire plus subtilement l'aspect et la texture même de la peau d'un serpent vivant, si unie, souvent bicolore, et si changeante d'aspect.

Cette ironie n'est plus de mise dans le plus sublime poème de Rimbaud, Faim, parmi les poèmes donnés en exemple de l' " alchimie du verbe ", au beau milieu de Délires II, dans Une saison en enfer. Un simple trait divise ce poème en deux suites de trois strophes de quatre vers. Cette disposition restitue pour les yeux du lecteur le sceau de Salomon, connoté par les « autels de Salomon » ${ }^{4}$ qui, dans la strophe 5, donneraient son sens aux contrastes sophistiqués des motifs disposés sur les deux moitiés du poème. Quand la mystique juive pointe son nez au sommet de l'expérience poétique de Rimbaud, ce " Jui[f] erran[t] de Norwège $\|^{5}$ (Comédie de la soif). En effet, la première moitié du poème se singularise par des valeurs sémantiques et des sonorités évoquant la dureté, tandis que la seconde vaut pour la douceur des valeurs et des « sons ». Ce partage, d'ailleurs nuancé, redonne vie à la définition hébraïque de la sagesse divine, dans laquelle s'entrelacent la Rigueur et la Douceur.

4 Rimbaud, Cuvres, op. cit., pp.266-7.

$5 \quad$ Ibid., pp. 199-201. 
La régularité des deux suites de douze vers que sépare un trait manifeste le sens mystique des « autels de Salomon ». Mais que penser du choix de l'impair pour les vers de sept pieds ? Ce choix de l'impair n'est d'ailleurs pas constant. Sa rigueur se tempère par de mystérieux écarts où se traduit, pas seulement pour nos yeux, le brisement de l'unité : celle des "vallées grises » ou des « cailloux qu'on brise ", où l'on peut voir une question angoissée à l'idéal exprimé par les mesures parfaites, indiquées dans la Bible, de « l'autel de Salomon ». Quoi qu'il en soit, le pluriel des « autels », comme celui des « Solognes » dans Michel et Christine, circonscrit le rapport de l'Un avec le multiple, le multiple dont la violence se poétise dans les détails environnant cette évocation.

S’il répond au projet du « voyant », ce phénomène dépasse le cadre de l'activité poétique. En 1891, la première note d'un bref itinéraire ou « Journal $»^{6}$ rédigé par le Rimbaud trafiquant, déjà frappé par la maladie qui l'emportera, nous fait sentir son déchirement intérieur, quand l'homme ne fait plus qu'un avec la civière qu'il a justement dessinée, moins bien sur un feuillet de cet itinéraire que dans le texte même de ce paragraphe dont l'agencement interne restitue en effet les lignes de la civière, devenue l'idéogramme du destin de Rimbaud.

Le mot «moitié »' dans la cinquième phrase de la première note du « Journal » en question, définit l'axe médian autour duquel se distribuent deux suites égales de termes. On songe au vers 26 de Comédie de la soif, " Divisez l'eau fine », qui divise en deux moitiés égales le dialogue versifié de cinquante-deux vers rapporté dans ce poème où Rimbaud fustige le démon de la symétrie, avec lequel son verbe ne fait qu'un.

La civière en effet se poétise dans la répétition, en symétrie dans le paragraphe, des noms propres de lieux. Plus alchimique, le rapport de la pluie et du vent dans la dernière phrase (« Nuit [...] toute le nuit »), avec ces mots notés dans la première moitié du paragraphe : « matin [...] matinMarécage ».

Mieux que les deux mentions de la « civière », l'alternance de la «Descente » et du mouvement inverse (« monter »), évoquent une recherche de l'équilibre, qui n'intéresse pas que le

$6 \quad$ Ibid., p. 766.

« Mardi 7 avril. / Départ du Harar à 6 h. du matin — Arrivée à Degadallal à 9 1/2 du matin. Marécage à Egou — Haut-Egou 12 heures - Egou à Balloua Fort 3 heures — Descente d'Egou à Ballaoua très pénible pour les porteurs qui s'écrasent à chaque caillou, et pour moi qui manque de chavirer à chaque minute. La civière est déjà moitié disloquée, et les gens complètements rendus - J'essaie de monter à mulet, la jambe malade attachée au cou - Je suis obligé de descendre au bout de quelques minutes et de me remettre en civière, qui était déjà restée un kilomètre en arrière. Arrivée à Ballaoua. Il pleut — Vent furieux toute la nuit. » 
corps de Rimbaud. Ce dernier n'a cure de la forme textuelle de ces notes, mais on peut souligner la suite de trente-six mots qui s'étire entre les deux mentions de la " civière », avant les dix-huit derniers mots de ce paragraphe. L'espace graphique restitue ainsi la « disloc[ation] » de la civière, en vertu d'une potentialité de l'écriture, jadis proclamée par le « voyant». On peut raffiner cette analyse, étendue à la syntaxe ou aux sonorités de certains mots. (Je l'ai mieux montré dans mon ouvrage Rimbaud malgré l'autre, 2014.)

Paul Claudel, premier éditeur de la correspondance africaine de Rimbaud, n'a jamais rapproché de la pensée de ce dernier sa vision des « idéogrammes occidentaux » où culmine le pouvoir de l'harmonie imitative. C'est pourtant à cette époque, dans la deuxième décade du XXe siècle, que Claudel honore la mémoire de Rimbaud dans Consécration, long poème en vers libres de quarantetrois distiques, présenté comme un triptyque centré sur le signe de l'étoile. Les huit derniers distiques reprennent les thèmes exposés dans la première partie, qui regroupe huit distiques. Or, l'image de Rimbaud mise en avant dans ce poème est celle du marcheur, puni de sa dromomanie par le retranchement de «L'équilibre de ces deux jambes en marche » ${ }^{7}$. Dans l'avant-dernier distique, cet énoncé reprend en écho le thème de la " marche ", dans le premier vers du poème : "La chose qui a mis Rimbaud en marche et qui l'a chassé de lieu en lieu toute sa vie »...

Plutôt que la marche, c'est le supplice des béquilles de l'amputé que restituent la composition en distiques, mais encore la présentation générale du poème. L'infirmité de Rimbaud revêt ainsi une valeur symbolique à l'égard du principe de tous les rythmes. (Cette intuition de Claudel se verra confirmée par un article fameux de Gérard Macé sur le destin de Rimbaud.) Le lecteur du poème partage donc physiquement, par le pouvoir suggestif de la matière écrite, les affres du dernier Rimbaud, restituées par le texte. Or, cette synesthésie s'élargit dans une révélation spirituelle, intéressant les zones les moins connues de notre esprit. - En accord avec l'unité du corps et de l'âme, proclamée dans la dernière phrase d'Une saison en enfer.

A la fin du premier volet du triptyque, les mentions du « Paradis » et de « l'Enfer » annoncent celle de Dieu : dans l'avant-dernier distique, et d'abord au dernier vers du volet central : «Quand Dieu même sur la croix pour nous s'est rompu et nous est ouvert? » Ce motif de la « croix » revient dans le 
texte, délimitant un espace de douze distiques : «J'en veux pour témoin cette croix, ayant fini d'écrire, que tu gravas sur ta table de travail. » Certes, ces mots ne sont pas soulignés pour parler à nos yeux, mais l'intérêt de Claudel pour l'aspect visuel des mots nous autorisent y à voir des signes parlants. D'autres motifs, comme celui de la "sœur », découpent l'espace graphique sur les mêmes mesures. La convergence du symbolisme de la croix et celui du nombre 12 (expression symbolique d'une idéale équité, envisagée par Claudel dans sa lecture de l'Apocalypse) se vérifie dans ces dispositions. Ces harmonies restituent pour les yeux du lecteur la fameuse croix, reconnue par Claudel visitant la maison de Rimbaud, que ce dernier aurait gravée sur la table où il écrivit Une saison en enfer. Le poème ne fait donc qu'un avec cette croix, sous les yeux ... ou sous l'ongle du lecteur, dont l'être même rejoint celui d'Arthur, à dix-huit ans comme à trente-six.

Claudel n'a pas attendu cette Consécration pour concurrencer le projet du « voyant », notamment en 1896 dans son poème Pagode (présenté comme une longue suite de paragraphes) où il relate sa visite d'une pagode chinoise qui, avec son temple et sa tour de sept étages, est une image architecturale de l'ordre cosmique, idéal avoué du poète Claudel. Le désir de transcrire dans son texte les sept étages de la tour se fait jour paragraphe 7, formé de cette seule phrase : «Il faut d'abord parler de la Pagode proprement dite $»^{8}$. Le détail chiffré de la première phrase du paragraphe 6 n'est pas moins suggestif : « J'ai devant moi la tour à sept étages ».

Claudel adapte, par un usage sophistiqué de la dispositio, l'étagement de son propos aux sept étages de la tour décrite. Les « sept étages » qui, dans le paragraphe 17, « sont une coupe de sept cieux mystiques », relaient à sept paragraphes de distance la vision, au paragraphe 10, de « deux dragons » qui « se disputent le joyau mystique », sur le faîte du toit.

J'ai étudié ailleurs ${ }^{9}$ les reprises en écho de certains détails architecturaux qui traduisent dans le texte même l'aspect de la pagode. Au paragraphe 8, les « tuiles noires formant des rainures profondes

$8 \quad$ Ibid., p. 26-30.

9 Voir M. Arouimi, « La Pagode graphique de Claudel », in Paul Claudel et la Chine, Wuhan University Press, 2010, p. 38-54. Il existe de nombreux antécédents critiques (et théoriques) à ma démarche Je songe aux travaux déjà anciens de Michel Butor, André Jolles, Iouri Lotman, Jean Rousset mais encore Anne-Marie Christin, et certains collectifs, comme les Cahiers Jussieu (1977, «L'espace et la lettre »), plus récemment Marking the Text : The Presentation of Meaning on the Literary Page (Cambridge University Press, 2000). Mais il s'agit le plus souvent de considérations générales, ou qui ne concernent que le jeu conscient des auteurs des textes cités. Le risque est grand, dans ma démarche, de la subjectivité du lecteur, dans une estimation des ressources visuelles de l'espace graphique de tel ou tel texte fameux. J'espère que la présente tentative donnera l'idée d'une méthode, qui requiert un regard simultané sur la forme et le contenu du texte. 
et de fortes côtes » ou, au paragraphe 13, les « rayons horizontaux » du soleil dans la " caisse sombre de la salle ", ces visions sont restituées par la simple alternance des paragraphes de longueur si inégale. Cinq d'entre eux, d'une ou deux phrases, et d'à peine deux lignes, strient l'espace graphique. Cet espace imite ainsi l'aspect extérieur du toit de la Pagode, de même que l'intérieur de la salle décrite au paragraphe 13. Le jeu de l'ombre et de la lumière, dans ces deux visions contrastives, illustre le Principe spirituel, matérialisé dans cet édifice.

Claudel emploie d'ailleurs un chiffre arabe en comparant la bouche d'un « gros poussah doré » à « un soupirail [qui] s'élargit aux coins comme un 8 ». Ce détail chiffré, au paragraphe 9, paraît signaler les calculs inconscients dont résulte l'écriture des huit premiers paragraphes. A l'inverse, vers la fin du paragraphe 11, " un garçon de douze ans » indiquerait la transition avec le paragraphe 12 : « Le dernier qui passe est un garçon de douze ans. Je gagne [...] la troisième cour. » Le mouvement du garçon et celui du visiteur ne font qu'un avec celui du scripteur, parvenu à une charnière de son édifice poétique. A son tour le lecteur s'identifie à ces actants, non sans partager l'ivresse probable des constructeurs de la pagode.

La récurrence de certains motifs, comme celui des yeux ou du ciel, mériterait un long commentaire. L' « œil unique » d'un mendiant au premier paragraphe et les « yeux fermés », plus fréquemment notés, évoquent le rapport de l'Un et du multiple. La scission de l'Un se poétise notamment dans le motif de la sonnette, au pluriel en 5, au singulier en 17 . Vers la fin du poème, la sonnette qui garnit « chaque angle de chaque toit » de la Tour est comparée à une «Syllabe lié » dont le « son inentendu » est celui de " chaque ciel ». Mieux que par les sonorités du verbe, ce son « inentendu » est rendu sensible par le rythme visuel et sonore de très nombreux termes, de valeur syntaxique variable. Le poème s'entend comme une amplification de la " formule conjuratoire ${ }^{10}$ évoquée par Claudel dans son Traité de la co-naissance à propos du simple emploi des noms, par lequel «nous nous substituons à la chose » désignée. Dans le paragraphe 9 de Pagode, l'évocation d'un « engin cylindrique pareil à un parasol fermé ou à un pétard », préfigure « l'engin rotatif » qui, dans un texte de 1910, désigne sous la plume de Claudel « le Yang et le Ying ». Claudel y voit «l'idée [bien avant Hegel] de l'identité des contradictoires $»^{11}$.

10 Paul Claudel, Euvre poétique, op. cit., p. 180.

11 Dans « Les superstitions chinoises », [Contacts et circonstances] CEuvres en prose, Bibliothèque de la Pléiade, 1965, p. 1081. 
Si synesthésie il y a, elle n'intéresse pas que l'immédiateté de nos sens. Claudel appliquera sa réflexion théorique sur ce phénomène en 1925, notamment dans sa Philosophie du livre. Le projet conscient du poète architecte, qui détient les secrets de l'architecture classique gothique, si proches de ceux de la perfection des reliures anciennes, se vérifie dans l'adéquation entre la beauté des édifices religieux évoqués dans cet essai et la construction sophistiquée de ce dernier. Claudel y cite d'ailleurs deux de ses poèmes, non moins dignes d'être rapprochés du bâti du « cloître avec ses deux colonnes et long de quatre pas $»^{12}$, évoqué avant la conférence proprement dite. Or le premier poème, de six vers et sans titre, composé au Japon, exprime « l'émotion » provoquée par un " mot », que fait résonner la cloche d'un temple. Le second poème, titré Religion du signe et composé en Chine, s'articule en deux moitiés : ses six premiers vers évoquent la singularité de l'écriture chinoise, et les six vers suivants décrivent un temple confucianiste, visité par Claudel.

Le syncrétisme des deux traditions, que je pourrais longuement montrer, vient corser l'interférence de l'architecture et de l'écriture, en prose ou en vers. La «conférence » proprement dite est introduite par quelques paragraphes, où sont mentionnées les « énormes courbes » du dôme de SaintPierre. Dans la première partie de sa conférence, Claudel évoque, intrigante symétrie, l' « énorme effort de tout un peuple qui de l'épaule essay[e] de faire craquer les Alpes $»^{13}$. Dans ce passage, les « stratifications » du destin du diplomate Claudel, trouvent leur pendant dans la dernière partie de l'essai, où les « stratifications d'une mine de charbon ${ }^{14}$ sont le comparant des richesses d'une bibliothèque. La matière écrite s'apparente ainsi aux « terrasses successives d'un grand jardin [japonais] $»^{15}$. Claudel suggère ainsi le principe commun qui se joue de la différence de la nature et de la culture, dans les visions matérielles où s'illustre ce principe. Cette révélation concerne notre être, tous sens confondus, comme le suggère le relais du ciel «bleu » de Florence, noté au début de la conférence, et « l'ouvrier dans son bleu » (souligné par Claudel) dans l'avant-dernier paragraphe. Cette reprise écho se lit comme une représentation des effets de synesthésie qui nous font littéralement toucher le secret du ciel, fût-il imité dans les œuvres d'art. Et si Claudel écrit dans cet essai : «L'amateur qui tourne les pages [des chefs d'œuvre poétiques de la renaissance italienne] n'a pas besoin de lire pour absorber le poème ", on peut suivre ce conseil en caressant du regard l'espace graphique de son essai, lui-même conçu comme

12 Claudel, Euvres en prose, op. cit., p. 68-81.

13 Ibid., p.70 et 71 .

$14 \quad$ Ibid., p. 70 et 78.

$15 \quad$ Ibid., p. 77. 
le lieu d'un « champ magnétique ${ }^{16}$ (suggéré à Claudel par le « rapport » du « bloc imprimé » et de la « marge »), qui touche tous les sens ou peu s'en faut.

Ce travail sur la langue peut évoquer celui de C.-F. Ramuz, à qui Claudel s'intéressa à diverses époques de sa vie ${ }^{17}$. Si pour Claudel, Ramuz est « un des meilleurs ouvriers de la langue », on doit observer que ce dernier, un peu comme Claudel qu'il n'imite pas, inflige à la syntaxe des distorsions qu'il justifie, dans une lettre fameuse de 1926 à son éditeur Grasset, par son désir de capter le phrasé des paysans du pays vaudois. Mais cet aveu en recouvre d'autres, concernant le désir, déclaré dans son Journal ou certains recueils de pensées, comme Remarques (rédigé au cours des années 20), de transposer dans ses textes, pour la faire éprouver par le lecteur, une entrave psychologique, dont Ramuz ressent l'emprise au fond de son être et qui, paradoxalement, détermine sa vocation littéraire, apparentée selon lui à celle d'un peintre.

J'ai étudié ailleurs, dans un article à paraître, l'analogie entre la technique des peintres qui sont l'objet des « critiques d'art » de Ramuz, et la facture de ses poèmes de jeunesse, où les bizarreries de la syntaxe, jointes à l'économie savante de la distribution des motifs, préfigurent plus qu'elles ne les imitent les recherches des peintres cubistes. Et l'auteur de ces poèmes se considère bien moins comme un poète que comme un peintre. Assez curieusement, les écrivains connus pour avoir plus ou moins consciemment partagé cette affinité avec les peintres - je songe à Kafka, féru d'estampes chinoises, mais encore à Tchekhov, ont en commun le projet violent d'agir sur les sens du lecteur, par une commotion justifiée comme le moyen d'un éveil spirituel.

Cette revendication picturale de Ramuz se fonde sur une conception de l'écriture qui coïncide avec celle de Rimbaud, modèle artistique et spirituel de Ramuz : quand le texte vaut par sa capacité

16 Ibid., p. 76.

17 Un texte majeur, constitué par divers écrits antérieurs, témoigne de cet intérêt, Du côté de chez Ramuz, publié en 1947 à l'occasion de la mort de Ramuz. 
de suggestion sensorielle, quasi visuelle, préférée aux valeurs mimétiques de la simple description ${ }^{18}$. Sans y voir une influence, l'usage que Ramuz fait du tiret, du moins dans un passage de son roman Présence de la mort, peut rappeler celui de Rimbaud dans Veillées II. Dans ce roman où sont d'ailleurs évoqués les effets délétères du mauvais mimétisme, un comptable regarde un paysage, assimilé à une page de ses comptes. Et cet homme « tire une barre [...] — tirant donc dans le ciel une barre au-dessus du pont $»^{19}$. Le narrateur qui rapporte cette vision est lui-même un écrivain, mais ce tiret, sous la plume de Ramuz, suggère son identification à ce comptable, dans une révélation que complètent maints détails du paysage illustrant la notion du « même ». Ramuz rejoint ainsi Rimbaud dans une remise en cause du Nombre, objet d'une figuration diversifiée dans la section III de Veillées, où s'enchaînent des symboles du mimétisme, au moins artistique.

Dans certains récits brefs de Ramuz, le jeu visuel et sonore de la lettre V, notamment à l'initiale de certains noms, comme le « Vanil » dans Le tout-vieux en 1910, exprime un aspect majeur du procédé littéraire, mieux poétisé en 1942 dans la nouvelle La foire. Sans parler d'influence, on peut encore rapprocher du garçon de douze ans « qui passe » dans le poème Pagode de Claudel, les deux protagonistes de La foire, que l'on voit « passe[r] » entre deux élévations rocheuses, au cœur même de cette nouvelle articulée en diptyque où deux histoires se voient raccordées ${ }^{20}$. Alors un simple espace blanc entre deux paragraphes paraît mimer celui qui sépare ces élévations. Cet espace figure aussi bien le moment où le lecteur, comme l'écrivain Ramuz lui-même, après le récit d'une première série d'événements, aborde le second versant de la nouvelle. Le lecteur éprouve simultanément l'effort de Ramuz et celui de ses personnages, qui exprime lui-même cet effort du poète architecte. Cet effet de miroir est rendu plus mystérieux par le drame de ces personnages qui sont un pitoyable avatar de l'Androgyne mythique, auquel réfère une symbolique imbibée de valeurs chrétiennes.

18 J'aurais aimé montrer que ce phénomène se retrouve chez d'autres écrivains, comme Scarron, dans un génial autoportrait littéraire où le « $\mathrm{Z}$ » auquel le poète compare son aspect physique, est encore la clef du zigzag sémantique et du rythme d'écriture qui, mieux que son évocation littérale, restituent cet aspect. De même chez Nerval, avec le portrait de l'escamoteur de La main enchantée. Les traits diaboliques du personnage, comparés à un «V $\mathrm{V}$, s'impriment dans l'imagination du lecteur avec une force qui est celle du texte du chapitre IV, à l'intérieur duquel des notations en chiffres romains suggèrent l'identification du texte, ou du pouvoir qui l'inspire, alchimique si l'on veut, au tourment sans âge dont les sourcils « dessinés comme un V » de l'escamoteur sont le chiffre. 
L'expérience poétique et le témoignage de Ramuz déplacent le sens des idéogrammes " occidentaux ». Ces derniers, tels que Claudel les présentent, risquent de nous apparaître comme autant de fantaisies, renouvelant le débat de l'arbitraire du signe. Dans La foire, la montagne ou le dos de la vieille, et les bretelles du patron d'un café, sont autant de figures du modèle intangible auquel se ramènent les formes de la création et celles de l'art. Ou figures du « sanglot de l'origine » dont parle Claudel dans son Traité de la co-naissance, autrement dit la division de l'être, qui détermine notre vision du monde?

Autre poète, qui fut un vrai peintre, Carlo Levi semble s'être efforcé de nous épargner, en écrivant Le Christ s'est arrêté à Eboli (1945), d'imaginer les peintures exécutées lors de son exil politique, objet de brèves allusions dans ce témoignage autobiographique. J'ai consacré un ouvrage ${ }^{21}$ à l'analogie de sa technique picturale et de sa technique littéraire, du moins dans ce récit. Curieusement, les couleurs privilégiées au fil du récit restituent pour le lecteur le drapeau abhorré de l'Italie fasciste. Mais cette transposition est bien moins raffinée que les sinuosités du style de Levi, adaptées au paysage décrit. Si le thème surabondant et diversifié des sonorités retient l'attention, c'est peut-être en raison des gammes lexicales soigneusement étagées dont le rythme, mystérieux par la rigueur de ses mesures, peut être ressenti comme une transposition de tous ces bruits, surtout bourdonnements, vibrations, etc. Autant d'échos dégradés du Verbe, principal objet du Cristo.

Le thème de la peinture (décors peints d'une représentation théâtrale, pitoyables imitations de l'activité du peintre par un très jeune garçon, etc.) révèle une intuition des mutations de l'art pictural, libéré de l'espace du cadre et de l'obligation d'imiter, même en le déformant, le réel. Les peintures réelles effectuées par Levi lors de cette expérience ne sont d'ailleurs pas si mimétiques. Au-delà de l'objet représenté, le peintre Levi manifeste un drame existentiel qui est aussi bien le sien que celui des autochtones : une division de l'être, justement interprétée dans la structure même du récit, tendu par des effets de symétrie et axé sur une allégorie géologique de cette division. (Un paysage orienté, décrit dans un passage dont l'organisation interne en traduit l'apparence...) 
L'idée d'une peinture en mutation, suggérée dans certains passages, est moins cruciale que celle d'un au-delà de la littéralité du texte écrit. Carlo Levi ne semble pas avoir calculé les dispositions textuelles qui révèlent ce procédé. Ce dernier est pourtant l'objet de maintes énigmes, empruntées au folklore populaire : formule inscrite sur une amulette, comptine à valeur thérapeutique. Autant de mises en abyme du projet littéraire de ce peintre considéré comme un mage par les autochtones, et qui était aussi médecin. Ces objets, ces bribes poétiques ne perdent rien de leur réalité en se transposant dans le récit tout entier, qui nous subjugue par son aura objectale.

L'intérêt tardivement déclaré de Levi pour Rimbaud suggère d'ailleurs la possibilité d'une influence, relative à la théorie du « voyant», dont certains aspects revivent dans les propos philosophiques de Levi. La pensée de ce dernier oscille entre la métaphysique et la violence interhumaine qui joueraient, l'une ou / et l'autre, un rôle inspirateur dans la maîtrise des formes, picturales ou littéraires, dont ces formules magiques sont la représentation. Le Cristo est en effet sous-tendu par une réflexion, girardienne avant la lettre, sur les schémas sacrificiels qui épongent une violence sans âge, liée au mimétisme interhumain. Certains artistes, qui connaissent dans cette violence le nerf de leur génie, n'en sont pas moins tentés, fugitivement pour Rimbaud, durablement pour Ramuz, de revendiquer une source plus spirituelle de la «magie » de leur écriture, cette magie qui s'aiguise dans les effets de synesthésie soulignés plus haut.

On m'excusera de constater ce partage sans le résoudre. L'influence de Rimbaud sur Claudel, mais encore sur Ramuz, et sur un autre plan, les raisons probables de l'intérêt de Carlo Levi pour Rimbaud, ne sauraient nous éclairer sur le phénomène étudié, qui échappe à l'influence littéraire. Je parlerai plutôt d'une communauté d'esprit qui se joue des différences culturelles et chronologiques, d'après mes recherches sur d'autres écrivains. Peut-être faut-il prendre au mot, malgré son humour apparent, l'auteur des Idéogrammes occidentaux. Et revenir sur le débat du Cratyle, que notre époque a refermé malgré l'avancée d'un Serge Boulgakov, dans sa Philosophie du verbe et du nom (1953). Quand le génie de ce grand linguiste s'emploie à cerner le pouvoir démiurgique du langage humain, un pouvoir hérité du Verbe, qui n'est pas un «mythe » pour cet auteur. 


\section{Ouvrages Cités}

AROUIMI, Michel. Magies de Levi. Fasano : Schena, 2006.

. «La Pagode graphique de Claudel ». in Paul Claudel et la Chine. Wuhan University Press, 2010.

BRAY, Joe, and Miriam HANDLEY, and Anne C. HENRY, eds. Ma(R)king the Text: The Presentation of Meaning on the Literary Page. Cambridge: Cambridge University Press, 2000.

CLAUDEL, Paul. CEuvre poétique. Bibliothèque de la Pléiade, 1967.

-. «Les superstitions chinoises». [Contacts et circonstances] CEuvres en prose. Bibliothèque de la Pléiade, 1965.

RAMUZ, C.-F. Romans II. Bibliothèque de la Pléiade, 2005.

—.Pastorale : Nouvelles et morceaux 1905-1946. Paris : Sang de la Terre, 1986.

RIMBAUD, Arthur. CEuvres Complètes. Bibliothèque de la Pléiade, 2009. 